\title{
Inflammation in Cardiovascular Disease
}

\author{
Chul Soo Park, MD \\ Division of Cardiology, Department of Internal Medicine, College of Medicine, The Catholic University of Korea, Yoido St. Mary's Hospital, Seoul, Korea
}

Refer to the page 383-391

There is robust evidence that $\mathrm{C}$-reactive protein (CRP) is a marker for cardiovascular disease (CVD) and mortality in patients at risk, as well as in patients with established CVD." AHA/ACC Guidelines recommend CRP for CV risk assessment in asymptomatic intermediate-risk men 50 years of age or younger or women 60 years of age or younger with a Ilb recommendation class. Recently, evidence has accumulated showing that the ratio of neutrophil to lymphocyte count (NLR) predicts cardiovascular diseases and is slowly emerging as an independent, useful prognostic parameter for CVD. ${ }^{2)}$ There have been some reports regarding NLR's possible role as a prognostic biomarker for CVD in Korean populations. Kim et al. ${ }^{3)}$ reported that diabetic patients with high NLR showed a greater prevalence of significant CAD compared to diabetics with low NLR. Nam et al. ${ }^{4}{ }^{4}$ reported that high NLR was associated with elevated coronary calcium in healthy males and Cho et al. ${ }^{5)}$ also reported that high NLR was an independent predictor for long-term adverse effects in patients undergoing percutaneous coronary intervention (PCI). The predictive superiority of NLR over inflammatory cells alone, such as WBC counts, may be due to many reasons including the fact that it is less likely to be influenced by various physiological conditions such as dehydration

Received: April 28, 2017

Accepted: May 5, 2017

Correspondence: Chul Soo Park, MD, Division of Cardiology, Department of Internal Medicine, College of Medicine, The Catholic University of Korea, Yoido St. Mary's Hospital, 62 Yoido-dong, Youngdeungpo-gu, Seoul 07345, Korea

Tel: 82-2-3779-1325, Fax: 82-2-3779-1374

E-mail:Charlie@catholic.ac.kr

- The author has no financial conflicts of interest.

This is an Open Access article distributed under the terms of the Creative Commons Attribution Non-Commercial License (http://creativecommons. org/licenses/by-nc/3.0) which permits unrestricted non-commercial use, distribution, and reproduction in any medium, provided the original work is properly cited. and exercise, even though these conditions may affect absolute number of individual cell types. Second and most importantly, NLR is a ratio of two different yet complementary immune pathways, thus integrating the deleterious effects of neutrophils, which are responsible for active nonspecific inflammation and lymphopenia, which are markers of poor general health and physiological stress.

Shin et al. $\left.{ }^{6}\right)$ proposed that a combination of CRP and NLR improved the prediction of all cause death compared to either CRP or NLR alone in patients with myocardial infarction. They analyzed 341 acute myocardial infarction (AMI) patients who underwent $\mathrm{PCl}$ and were followed up for 24 months. A multivariate Cox regression analysis showed high NRL and CRP were independent predictors of all cause death with a hazard ratio of 23.17 (see Table 4). However, given that NLR and CRP have been known as useful biomarkers to predict cardiovascular death in various patient populations, this result is no surprise. The sensitivity and specificity of biomarkers should be evaluated using the receiver operating characteristic (ROC) area under curve (AUC). Therefore, increments in AUC using a combination of CRP and NLR should be investigated compared to those of CRP and NLR alone to demonstrate the usefulness of combining these 2 biomarkers for risk prediction. However, the AUC for NLR and CRP was shown to be 0.609 and 0.636 respectively, while the AUC for combined NLR and CRP has not been investigated. Furthermore, if there is an AUC increment when NLR and CRP are combined, the investigators should test whether the addition of NLR provided a prediction increment over CRP or NLR alone using an integrated discrimination index or net reclassification index.

There are some limitations in this article including a small sample and statistical reliability. However, this article reconfirmed that inflammation, in particular alteration of leucocyte function, maybe an important pathophysiologic pathway in CVD and raises the possibility that anti-inflammatory drugs could be used to treat or prevent myocardial infarction. Even though inflammatory pathways as discussed in this article remain tempting targets for therapeutic intervention, several observations and trials have been disappointing. Non-steroidal anti-inflammatory agents, in particular cyclooxygenase-2 (COX-2) selective inhibitors, did not 


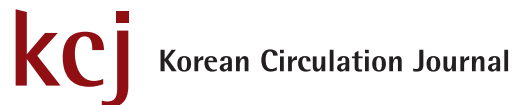

improve cardiovascular outcomes, and even posed a cardiovascular risk.7) The COX-2 selective inhibitors may change the balance between thromboxane $A 2$ and prostacyclin production toward thromboxane A2 production which causes platelet aggregation, thrombus formation and vasoconstriction. Steroids have potent and widespread anti-inflammatory effects, but their use may result in numerous side effects including hypertension, insulin insensitivity, and obesity. Aspirin provides cardiovascular protection. But, because the doses of aspirin that exert beneficial cardiovascular effects fall far below the doses required for a true anti-inflammatory effect, aspirin's benefit maybe due to other mechanisms than anti-inflammation. Meanwhile, statin and colchicine ${ }^{8)}$ which are anti-inflammatory agents used in rheumatoid arthritis, were associated with an impressive reduction in cardiovascular events, but the exact mechanism of their cardiovascular protection remains controversial. There are still ongoing trials using new antiinflammatory agents such as MTX and anti IL-1beta. Interestingly, losmapimod which targets p38 MAP kinase, a key participant in the control of inflammation, did not reduce cardiovascular events in patients with myocardial infarction despite reducing CRP.9)

In conclusion, this article suggested using NLR, a new biomarker, along with the well-known CRP, because inflammation is a major determinant of CVD. However, studies exploring pivotal inflammatory steps or pathways linked with CVD should provide a new path forward for the therapeutic application of antiinflammatory agents in CVD.

\section{References}

1. Ridker PM. C-reactive protein and the prediction of cardiovascular events among those at intermediate risk: moving an inflammatory hypothesis toward consensus. J Am Coll Cardio/ 2007;49:2129-38.
2. Horne BD, Anderson JL, John JM, et al. Which white blood cell subtypes predict increased cardiovascular risk? J Am Coll Cardiol 2005;45:1638-43.

3. Kim BJ, Cho SH, Cho KI, Kim HS, Heo JH, Cha TJ. The combined impact of neutrophil-to-lymphocyte ratio and type 2 diabetic mellitus on significant coronary artery disease and carotid artery atherosclerosis. J Cardiovasc Ultrasound 2016;24:115-22.

4. Nam SH, Kang SG, Song SW. The neutrophil-lymphocyte ratio is associated with coronary artery calcification in asymptomatic Korean males: a cross-sectional study. Biomed Res Int 2017;2017:1989417.

5. Cho Kl, Ann SH, Singh GB, Her AY, Shin ES. Combined usefulness of the platelet-to-lymphocyte ratio and the neutrophil-to-lymphocyte ratio in predicting the long-term adverse events in patients who have undergone percutaneous coronary intervention with a drugeluting stent. PLoS One 2015;10:e0133934. doi:10.1371/journal. pone.0133934. eCollection 2015.

6. Shin HC, Jang JS, Jin HY, et al. Combined use of neutrophil to lymphocyte ratio and c-reactive protein to predict clinical outcomes in acute myocardial infarction patients undergoing percutaneous coronary intervention. Korean Circ J 2017:47;383-91.

7. Gislason G, Jacobsen S, Rasmussen J, et al. Risk of death or reinfarction associated with the use of selective cyclooxygenase-2 inhibitors and nonselective nonsteroidal antiinflammatory drugs after acute myocardial infarction. Circulation 2006;113:2906-13.

8. Nidorf SM, Eikelboom JW, Budgeon CA, Thompson PL. Low-dose colchicine for secondary prevention of cardiovascular disease. J Am Coll Cardiol 2013;61:404-10.

9. O'Donoghue ML, Glaser R, Cavender MA, et al. Effect of losmapimod on cardiovascular outcomes in patients hospitalized with acute myocardial infarction: a randomized clinical trial. JAMA 2016;315:1591-9. 